\section{Cahiers de Narratologie}

Analyse et théorie narratives

$10.1 \mid 2001$

La voix narrative

\title{
Les rapports entre la voix et le point de vue dans les récits de voyage de Camillo José Cela
}

\section{Carmen Dubois}

\section{(2) OpenEdition \\ 1 Journals}

\section{Electronic version}

URL: http://journals.openedition.org/narratologie/6971

DOI: 10.4000/narratologie.6971

ISSN: 1765-307X

\section{Publisher}

LIRCES

\section{Printed version}

Date of publication: 1 January 2001

Number of pages: 387-395

ISBN: 2914561032

ISSN: 0993-8516

\section{Electronic reference}

Carmen Dubois, "Les rapports entre la voix et le point de vue dans les récits de voyage de Camillo José Cela", Cahiers de Narratologie [Online], 10.1 | 2001, Online since 14 November 2014, connection on 23 February 2021. URL: http://journals.openedition.org/narratologie/6971 ; DOI: https://doi.org/ 10.4000/narratologie.6971 


\title{
LES RAPPORTS ENTRE LA VOIX ET LE POINT DE VUE DANS LES RÉCITS DE VOYAGE DE CAMILO JOSÉ CELA
}

\author{
Carmen DUBOIS
}

L'interrogation sur l'existence de règles narratologiques spécifiques aux récits de voyage a donné lieu à des réponses fort diverses, voire opposées.

Un certain nombre de critiques nient l'existence même du récit de voyage en tant que genre autonome. C'est le cas de Jean-Luc Moreau lorsqu'il affirme que puisque «il y a des voyages de toutes sortes, il y a aussi toutes sortes de récits de voyage ${ }^{1}$. Les possibilités narratologiques étant presque infinies, il conclut à l'impossibilité d'élaborer une théorie de ce type de récit..

D'autres critiques ont tenté d'isoler des traits constitutifs. Ainsi, Adrien Pasquali ${ }^{2}$ signale l'existence d'une évolution narratologique, à savoir: le narrateur-personnagetémoin des plus anciens récits de voyage aurait laissé place, dans la tradition la plus proche, à un narrateur-personnageacteur. Carlos García Romeral Pérez ${ }^{3}$ parle, quant à lui, de la prédominance de la première personne et de la coïncidence entre le narrateur et le voyageur. Juan Francisco Villar Dégano ${ }^{4}$ désigne comme élément définitoire l'emploi du « je »

${ }^{1}$ Jean-Luc MOREAU, « Odyssées », Ecrire le voyage, Textes réunis par György Tverdota, Tours, Presses de la Sorbonne Nouvelle, 1994, p. 38.

2 Adrien PASQUALI, Le tour des horizons. Critique et récits de voyages, Paris, Klincksieck, 1994.

3 Carlos GARCIA-ROMERAL PEREZ, Bio-bibliografía de viajeros españoles (1900-1936), Ollero Ramos Editores, Madrid, 1997.

4 Juan Francisco VILLAR DEGANO, « Paraliteratura y libros de viajes » in Libros de viajes, Murcia : Universidad de Murcia, 1996. 
en tant que fil conducteur de l'expérience personnelle du voyageur qui recouvre avec sa vision une géographie qui lui est étrangère.

Bien que la terminologie employée par les critiques soit fort hétérogène, la question de l'instance narrative et de la perspective dans les récits de voyage semble jouir d'un certain consensus. Tenant compte des remarques précédentes, nous pouvons retenir comme constantes narratologiques des récits de voyage la prédominance d'un narrateur homodiégétique - le plus souvent autodiégétique - et la focalisation interne - le personnage focal étant le plus souvent le voyageur.

Examinons désormais si Camilo José Cela, le long de sa nombreuse production voyageuse - il a écrit huit livres de voyage - a adopté ces modèles.

Si l'on considère le long et complexe processus d'écriture des récits de voyage de l'auteur, l'on découvre que le choix de l'instance narrative, loin d'être une décision facile, est l'aboutissement d'un cheminement émaillé d'hésitations. Les cahiers de notes - qui constituent l'embryon, la première esquisse des futurs récits - témoignent des doutes de l'écrivain qui oscille entre un narrateur homodiégétique et un narrateur hétérodiégétique. Voilà les premières annotations de son premier parcours à travers la Alcarria : « De repente, al escapar de la marquesina, el viajero descubre que ya es de día ». Quelques pages plus loin, l'instance narrative est modifiée : "Salimos de Guadalajara. Me lavo en un regato a la salida de Guadalajara y como en una vaguada, en un olivar ». Au narrateur absent de l'histoire s'est substitué un narrateur autodiégétique qui raconte sa propre expérience. Dans les autres carnets de voyage nous retrouvons systématiquement ce deuxième type de narrateur. Ainsi, l'écrivain note lors de son voyage à travers les Pyrénées de Lérida : "Duermo en Caldas de Bohí »; dès les premières annotations de son dernier périple - où il sillonne à nouveau la Alcarrria ce narrateur autodiégétique prend en charge le récit : «Un motorista muy espectacular se mete con Oteliña y yo, por señas, me cago en su padre. »

Dans les manuscrits - qui représentent l'étape suivante du processus d'écriture - l'écrivain revient à un narrateur 

de voyage de C.-J. Cela

absent de l'histoire ; il adoptera définitivement cette modalité narratologique, l'appliquera à l'ensemble des textes de voyage et il restera fidèle à ce choix le long des huit livres de voyages.

Comment expliquer cette transformation au niveau de la personne, cette conversion de l'homodiégétique à l'hétérodiégétique ? C.-J. Cela lui-même nous apporte un premier élément de réponse; dans la préface au quatrième tome de son Obra Completa, "Con la mochila al hombro y una paz infinita en el corazón », il déclare :

El vagabundo - que ahora habla de él como de otro hombre, objetivándose en la distanciadora y persuasiva tercera persona $[\ldots]^{5}$

Ce serait donc le souci d'objectivité qui aurait poussé l'écrivain à opérer ce changement d'instance narrative. Il est vrai que le narrateur extradiégétique introduit une instance supplémentaire entre l'histoire et le récit qui s'en trouvent de cette façon distanciées. Le lecteur n'a plus la vision directe du témoin-voyageur mais celle d'un narrateur étranger à l'histoire. La subjectivité du narrateur autodiégétique se trouve ainsi substituée par la froide neutralité d'une instance extérieure. L'impression d'immédiateté du récit s'affaiblit mais l'effet d'impartialité en sort renforcée : l'instance narrative n'étant pas impliquée dans les événements racontés, le risque de subjectivité est, à priori, écarté.

Cependant, ce prétendu détachement du narrateur n'est qu'illusoire et il s'avère en totale discordance avec le point de vue adopté. En effet, comme le signale à juste titre Gérard Genette, le récit impersonnel tend à la focalisation interne par discrétion et respect de ses personnages; nous avons vu aussi que les récits de voyage adoptaient généralement ce type de focalisation.

Lorsque que nous considérons l'ensemble des livres de voyage de C.-J. Cela, nous ne trouvons que quelques

5 Camilo José CELA. "Con la mochila al hombro y una paz infinita en el corazón », Viajes por España, 1. (1948-1952). Barcelona : Ediciones Destino, 1965, p. 10. 
passages descriptifs dans lesquels la perspective narrative coöncide avec la vision du vagabond. C'est le cas de ce court passage, extrait de Judios, moros y cristianos, où le lecteur découvre la ville de Ecija à travers le regard du vagabond :

En Ecija, el vagabundo vio muy nobles palacios y muy bellas torres. ${ }^{6}$

Ce type de focalisation - qualifiée d'interne - est assez restrictif dans le sens où le narrateur ne peut raconter que ce que « sait » ou « voit » le personnage focal. Elle ne convient pas au tempérament de l'auteur qui lui préfère une perspective lui permettant de contrôler davantage son œuvre. Le cadre imposé par un narrateur extradiégétique se révèle trop étroit pour rendre compte d'un contenu narratif qui va bien au-delà de la simple relation de voyage. Pour suppléer cette incapacité, il aura recours à un narrateur susceptible de se rendre maître d'une expérience objectivée, de gérer l'émission de l'information et d'imposer une certaine lecture de son œuvre. Le point de vue dominant correspond ainsi à celui d'un narrateur omniscient qui voit tout et sait tout. Il connaît par avance l'itinéraire que le vagabond va suivre ; ainsi, nous lisons dans un passage de Primer viaje andaluz:

En Navarte, de nuevo en el camino de Santesteban, $a$ donde no llegará el vagabundo, luce, sobre la fachada de un chalet, el escudo de Navarra hecho con verde y cuidadosa enredadera, con trepadora amable y delicada. ${ }^{7}$

S'il est au courant de la direction que le vagabond va prendre, le narrateur n'ignore pas non plus ses pensées, ses sentiments, ses états d'âme.

Il est dans le secret des bonnes intentions qui poussent le voyageur à prendre la route :

El viajero va lleno de buenos propósitos : piensa rascar el corazón del hombre del camino, mirar el alma de los caminantes asomándose a su mirada como al brocal de un

6 Camilo José CELA. Primer viaje andaluz, Viajes por España, 3. (1959-1964). Barcelona : Ediciones Destino, 1968, p. 205.

7 Camilo José CELA. Primer viaje andaluz, Viajes por España, 3. (1959-1964). Barcelona : Ediciones Destino, 1968, p. 31-32. 
Les rapports entre la voix et le point de vue dans les récits de voyage de C.-J. Cela

pozo. Tiene buena memoria y quiere deshacerse de la mala intención, como de un lastre, al dejar la ciudad. ${ }^{8}$

Il semble partager la fatigue physique du héros :

Está cansado y nota un alivio grande dejando caer las piernas, como marionetas, en la primera postura que quieran encontrar. ${ }^{9}$

On ne peut donc pas conclure avec José M. Pozuelo Yvancos à l'existence d'un point de vue béhavioriste où «el ser, el estar, el hacer predominan sobre el parecer o el juzgar ${ }^{10}$. En réalité, le savoir est celui du narrateur, pas celui du vagabond qui garde ainsi une vision naïve et fraîche. Et il s'agit d'un savoir que le narrateur se complaît à proclamer.

Quoique absent de l'histoire qu'il raconte, ses interventions dans le récit sont innombrables : il s'érige en commentateur éclairé des actions et des situations. Les sujets traités par le narrateur sont fort variés - nous trouvons des digressions historiques, géographiques, linguistiques, artistiques, métaphysiques - mais le ton est très souvent didactique. Cette amplification de la fonction idéologique du narrateur peut être considérée comme l'empreinte de l'autorité souveraine de l'écrivain. La présence du narrateur - perceptible dès le premier récit - ne fait que s'accentuer dans les textes postérieurs au point de pouvoir affirmer avec Angel Iglesias Ovejero que « el narrador se instala como verdadero personaje principal. $» 11$

Le narrateur use et abuse de sa fonction idéologique ; l'essai envahit le récit de voyage qui en sort métamorphosé.

${ }^{8}$ Camilo José CELA. Viaje a la Alcarria, Viajes por España, 1. (1948-1952). Barcelona : Ediciones Destino, 1965, p. 32.

9 Camilo José CELA. Viaje a la Alcarria, Viajes por España, 1. (1948-1952). Barcelona : Ediciones Destino, 1965, p. 29.

10 Jose M. POZUELo YVANCOS, "Introducción a Viaje a la Alcarria ». Madrid : Espasa Calpe, 1996, p. 24.

11 Angel IGLESIAS OVEJERO, «Cela onomaturgo : los nombres propios en el gallego y su cuadrilla "Revista Hispanistica. Université de Bourgogne, 1991, p. 85. 
Toutefois, les interventions du narrateur dans le récit ne se limitent pas aux digressions classiques que l'on vient de signaler ; emporté par sa verve intarissable, il va jusqu'à se dédoubler en narrataire afin d'assouvir cette passion des mots.

Une joyeuse troupe de narrataires fait ainsi irruption dans le récit; il s'agit d'auditeurs anonymes - désignés par des étiquettes telles « usted », "señor », "señora »- et éberlués qui interrompent le narrateur pour le questionner, énoncer une bêtise, le contredire. La présence de ces narrataires est très discrète dans les premiers récits ; progressivement leurs interventions se multiplient et finissent par envahir le récit. Le rythme de la narration est entrecoupé par des petits dialogues - une ou deux répliques - entre narrateur et narrataire anonyme dont le rôle est fort varié.

Ils peuvent illustrer ou compléter une idée précédemment exposée par le narrateur. A titre d'exemple l'on peut citer le thème de la critique du progrès qui, amorcé par le narrateur, est développé dans un dialogue très savoureux :

La mugre del subdesarrollo está más hecha a la medida del hombre que el detergente del progreso, que se ciñe mejor a la horma de las máquinas y demás ingenios que aherrojan y aplastan y humillan al hombre.

- ¿Usted prefiere una cabra a un transistor?

- No lo dude ; las cabras son animalitos que igual valen para un roto (matar al hambre, por ejemplo) que para un descosido (tener el apetito venéreo desmandado y no dar con una vagina de la misma especie a su debido tiempo, pongamos por caso). Las cabras son bestezuelas de mucha utilidad y compañía : no hace falta llegar hasta Amaltea, la cabra que amamantó a Júpiter a sus pechos, para darse cuenta de que esto es verdad.

- ¡ Hay que joderse, qué cultura !

- Por favor, déjeme usted continuar. Ahí tiene usted al Simeón Corcuera, el sacristán de Ocentejo, que desde que se quedó viudo hace vida marital con la cabra Algazula; el sacristán y su coima, aunque ésta sea 
Les rapports entre la voix et le point de vue dans les récits

de voyage de C.-J. Cela

animal irracional, se entienden en lengua migaña y son muy felices.

- Ya, ya...12

Les intrusions sont parfois très utiles au narrateur qui s'en sert pour reprendre le fil de l'histoire ou aménager une transition entre deux sujets différents.

Dans Viaje al Pirineo de Lérida, ce dialogue entre narrateur et narrataire, placé après un long et érudit paragraphe consacré à l'histoire des contes de Pallars, anime le récit et crée un effet humoristique par le contraste thématique et de ton :

- A mí me gustaría tener un loro que supiera cantar en francés como los poetas de la Edad Media, que eran unos golfos gloriosos: on se fait putain comme on se fait nonne... En inglés arcáico o, quizás mejor, en viejo slang, puta y monja de decía de la misma manera : núm. Villón, de haber sido inglés, hubiera podido entretenerse con muy hermosos juegos de palabras. Hamlet, el descarado, le grita a Ofelia : Get thee to a nunnery, vete a un prostíbulo. Y Astrana, que era de la provincia de Cuenca, y antes Moratín, traducen nunnery por convento. ; Así da gusto !

- Pues a mí lo que me gustaría era tener amores con una señora gorda y cariñosa y teñida de rubio, que hubiera heredado de un tío cura un molino maquilero. ${ }^{13}$

Nous pouvons affirmer que le narrateur celien est décidément orienté vers lui-même et vers son propre travail d'écriture qu'il n'hésite pas à porter en dérision dans ce dialogue avec un narrataire anonyme :

- ¿Por qué no habla usted más claro ?

- Porque no me da la gana, gentil señorita, sazonado bonbón, tía buena; esto de los sermones en clave, es

12 Camilo José CELA. Nuevo viaje a la Alcarria. Barcelona : Plaza \& Janés Editores, 1994, p. 25.

13 Camilo José CELA. Viaje al Pirineo de Lérida, Viajes por España, 3. (1959-1964). Barcelona : Ediciones Destino, 1968, p. 392. 
algo que se nos da muy bien a los patriarcas. ¿Quiere usted que echemos un pasodoble ? $^{14}$

$* * * *$

Nous avons montré que si Camilo José Cela avait opté, dans une première phase d'écriture, pour un narrateur homodiégétique ce n'était que pour mieux accomplir une conversion, tardive mais définitive, de l'autodiégétique à l'hétérodiégétique. Nous avons signalé ensuite que, paradoxalement, la perspective adoptée correspond à l'omniscience du romancier classique et non pas à la focalisation interne traditionnelle dans les récits de voyage.

Si l'écrivain renverse les conventions narratologiques du genre, c'est parce qu'il aime à rester maître de son œuvre. En effet, il est, en dernière instance, celui qui a le savoir : il sait ce qu'il va raconter, il possède l'information narrative. Il détient aussi le pouvoir: il décide comment réguler cette information à travers le choix d'une instance narrative et d'une perspective déterminées, en l'occurrence un narrateur extérieur à l'histoire et omniscient. Usant de ses pouvoirs, l'auteur va contrôler l'information et nous la livrer selon les modalités qui semblent être les plus adaptées à sa conception du projet artistique. C.-J. Cela nous propose ainsi un nouveau type de récit de voyage dont les règles restent à déchiffrer.

14 Camilo José CELA. Viaje al Pirineo de Lérida, Viajes por España, 3. (1959-1964). Barcelona : Ediciones Destino, 1968, p. 649. 


\section{BIBLIOGRAPHIE}

a) Sources primaires (œuvres de Camilo José Cela étudiées)

1. CELA (Camilo José). - Obra Completa. Tomo IV. Barcelona : Destino, 1965. - 624 p.

2. CELA (Camilo José). - Obra Completa. Tomo V. Barcelona : Destino, 1966. - 484 p.

3. CELA (Camilo José). - Obra Completa. Tomo VI. Barcelona : Destino, 1968. - $706 \mathrm{p}$.

4. CELA (Camilo José). - Nuevo viaje a la Alcarria. Barcelona : Plaza \& Janés, 1994. - 266 p. - (Ave Fénix ; $n^{\circ} 171$.)

b) Sources secondaires

1. Libros de viajes: actas de las Jornadas sobre "Los libros de viaje en el mundo románico ", celebradas en Murcia del 27 al 30 de noviembre de 1995 / sous la direction de Fernando Carmona Fernández y Antonia Martínez Pérez. - Murcia : Universidad de Murcia, 1996. $343 \mathrm{p}$.

2. CARRIZO RUEDA ( Sofía). - Poética del relato de viajes. - Kassel : Reichenberger, 1997. - 187 p. (Problemática literaria ; 37.)

3. GARCIA BERRIO (Antonio), HUERTA CALVO (Javier). - Los géneros literarios: sistema e historia (Una introducción). - 2e éd. - Madrid : Cátedra, 1992. - 276 p. (Crítica y estudios literarios.)

4. GARCIA-ROMERAL PÉREZ (Carlos). - Bio-bibliografía de viajeros españoles por España y Portugal (19001936). - Madrid : Ollero y Ramos, 1997. - 261 p. 